\title{
The radiation-induced chemistry in solid xenon matrices
}

\author{
V.I. Feldman ${ }^{1}$, A.V. Kobzarenko ${ }^{1}$, A.Y. Orlov ${ }^{2}$, and F.F. Sukhov ${ }^{1}$ \\ ${ }^{1}$ Department of Chemistry, M.V. Lomonosov Moscow State University, Moscow 119991, Russia \\ E-mail: vladimir.feldman@rad.chem.msu.ru \\ ${ }^{2}$ Institute of Synthetic Polymer Materials, 70 Profsoyuznaya Str., Moscow 117393, Russia
}

Received May 11, 2012

\begin{abstract}
The paper presents an overview of recent studies on the radiation-chemical transformations of guest molecules in solid xenon induced by fast electrons and x-ray irradiation. Specific features of the experimental approach based on combination of matrix isolation IR and EPR spectroscopy are briefly outlined (with particular impact on using monoisotopic and isotopically enriched xenon matrices). The results reveal rich and diverse radiation-induced chemistry in solid xenon, which is considered in the following major aspects: (1) matrix-induced and matrix assisted transformations of the primary guest radical cations; (2) production and dynamics of hydrogen atoms; (3) formation of xenon hydrides. Finally, preliminary results on the radiation-induced generation of oxygen atoms and ions in solid xenon are presented.
\end{abstract}

PACS: 82.50.-m Photochemistry;

82.33.Pt Solid state chemistry;

82.30.Cf Atom and radical reactions; chain reactions; molecule-molecule reactions;

82.30.Fi Ion-molecule, ion-ion, and charge-transfer reactions.

Keywords: matrix isolation, radiation chemistry, xenon, radical ions, hydrogen atoms, noble-gas hydrides.

\section{Introduction}

The photochemical transformations induced by UV radiation of isolated guest molecules in solid noble gas matrices were extensively investigated during several decades. The results of these studies constitute an important part of modern basis of solid-state photochemistry and matrix isolation spectroscopy $[1,2]$. Meanwhile, the corresponding processes induced by high-energy radiation (usually referred to as radiolysis) are still poorly understood. The basic difference between photolysis and radiolysis, as applied to matrix isolation, results from different mode of energy absorption. Indeed, the UV irradiation (photolysis) leads to selective excitation of the guest molecules, so the primary photoprocesses in matrices are usually similar to those known in the gas phase. The matrix mainly acts as a reservoir (or "cooling bath"), which may affect the energy dissipation and separation of reaction products. In contrast, the high-energy radiation is absorbed by matrix resulting in formation of mobile positive holes, excitons and electrons, which may reach the guest molecules acting as defects. In this case, the matrix properties should crucially affect the probability of "activation" (ionization or excitation) of an isolated molecule and the population of its electronic states, so, in general, the primary processes cannot be adequately simulated in the gas phase.
Recent extensive studies of charge migration and trapping in irradiated solid noble gases doped with simple molecular impurities performed by Savchenko and co-workers [3-6] revealed important features of physical aspects of the radiation-induced processes. Regarding the radiationinduced chemistry, the situation appears to be more complicated. The formation of radicals from simple guest molecules upon irradiation in solid noble gases has been demonstrated some fifty years ago [7]. This result actually reflects effective charge and/or excitation transfer from noble gas hosts to solutes, which was used for generation of matrix isolated radicals and radical ions characterized mainly by EPR spectroscopy [8-12]. However, the mechanistic aspects were not studied in detail. During the past fifteen years we performed systematic studies of the effects of high-energy radiation on organic molecules isolated in different noble-gas hosts using a combination of IR and EPR spectroscopy [13-22]. These experiments provided quantitative estimates for overall efficiency of the radiation-induced degradation of guest molecules in terms of radiation-chemical yields and made it possible to propose a general formal scheme of chemical transformations:

$\mathrm{Ng} \rightarrow \mathrm{Ng}^{+} \cdot+\mathrm{e}^{-}$(primary ionization),

$\mathrm{Ng}^{+} \cdot+\mathrm{RH} \rightarrow\left(\mathrm{RH}^{+}\right)^{*}+\mathrm{Ng}$ (positive hole transfer), 
$\left(\mathrm{RH}^{+}\right)^{*} \rightarrow \mathrm{RH}^{+\cdot}$ (relaxation),

$\left(\mathrm{RH}^{+}\right)^{*} \rightarrow$ products (high-energy ionic channels),

$\mathrm{RH}^{+} \cdot+\mathrm{e}^{-} \rightarrow \mathrm{RH}^{*}$ (ion-electron recombination),

$\mathrm{RH}^{*} \rightarrow \mathrm{R}^{\cdot}+\mathrm{H}^{\cdot}$ (homolytic dissociation of excited molecules),

$\mathrm{RH}^{*} \rightarrow$ non-radical products (other dissociation channels for $\left.\mathrm{RH}^{*}\right)$.

Here $\mathrm{Ng}=\mathrm{Ar}, \mathrm{Kr}$ or $\mathrm{Xe}$ and $\mathrm{RH}$ denotes a guest organic molecule. Certainly, this scheme is not a complete description of the radiation-induced processes in matrices (it disregards, for example, "self-trapping" of mobile matrix holes and electrons, which may be especially important for xenon). However, if we focus on the fate of the guest molecules, it works well for different-type moderate-size RH species with relatively low ionization potentials (IP $<10 \mathrm{eV})$.

As follows from this scheme, the principal pathway of "activation" of the guest molecule is positive hole transfer from matrix (3). This conclusion is supported well by the effect of electron scavengers, which terminates the ionelectron recombination (5). It was shown that the addition of electron scavengers resulted in drastic suppression of formation of $\mathrm{H}$ atoms produced by the ion-electron recombination followed by homolytiyc dissociation of neutral excited states (6) and, in certain cases, gave high yields of the trapped radical cations $\mathrm{RH}^{+} \cdot[14,16,20,23]$. Meanwhile, it is worth noting that the positive hole transfer (3) is typically a highly exothermic process (in a crude approximation, the excess energy can be deduced from the gap in the IP values between matrix atom and guest molecule, which varies from 2 to $7 \mathrm{eV}$ ) [16]. For this reason, the guest radical cation is formed in an excited state (which may imply both electronic and vibrational excitation), so it may undergo rapid fragmentation of rearrangement (4), competing with relaxation (3). Experimental indications of occurrence of the high-energy ionic channels (particularly important in argon with the highest IP value) were reported in early studies [10,24,25]. Detailed investigations revealed a rather strong and complex effect of molecular structure on the relaxation of excited radical cations in a solid argon matrix $[17,19,22]$. On the other hand, the matrix polarizability was found to be an important factor, which may control the reactivity of the radiation-induced ionic species $[16,18]$. As a whole, our studies on radiation-induced transformations of organic molecules in solid noble gas matrices revealed a diverse chemistry, which is essentially a matter of interplay between different electronic characteristics of the matrix used [16,17,20].

Among the noble gas media, xenon is often considered as the worst matrix host for spectroscopic studies of isolated species. Indeed, relatively high polarizability of xenon results in strong interaction with guest species (in par- ticular, for ionic intermediates), which may lead to considerable broadening of the IR absorptions. Furthermore, high abundance of magnetic isotopes with non-zero nuclear spin in natural xenon causes strong broadening of the EPR signals from radicals and radical ions trapped in a solid xenon matrix. Nevertheless, from the point of view of low-temperature chemistry, solid xenon is a promising host, because it offers much wider range of temperature stability in comparison with other noble gas matrices. Particular interest to this field in the past fifteen years is associated with discovery of a novel family of unusual molecules, noble gas hydrides $\mathrm{HNgY}$ (Ng represents a noble gas atom and $\mathrm{Y}$ is an electronegative atom or fragment) [26]. This family now includes more than twenty molecules and the majority of them are xenon compounds [27]. The common way of preparation of xenon hydrides includes dissociation of an appropriate precursor in a solid xenon matrix below $20 \mathrm{~K}$ followed by an annealinginduced mobilization of $\mathrm{H}$ atoms, which occurs at ca. $40 \mathrm{~K}$ $[23,28,29]$. Most of these species were first obtained in the University of Helsinki using photolysis for dissociation of precursor molecules (see, e.g., Ref. 30 for more detailed description). On the other hand, we have shown [31] that using high-energy radiation also allows one to obtain high yields of xenon dihydride from different precursors and this method was applied for preparation of a number of HXeY molecules [32,33].

This paper presents an overview of our recent studies on various aspects of the radiation-induced chemical transformations of guest molecules in solid xenon. In addition, we report some new results and discuss the prospects of this fascinating field at the interface of low-temperature physics and chemistry.

\section{Experimental methods}

\subsection{Common features of the experimental procedure}

Basic features of our experimental set-ups used for combined spectroscopic studies of the radiation induced transformations of matrix isolated molecules by IR and EPR spectroscopy were described elsewhere [14,15,22]. Original continuous-flow helium cryostats used in earlier experiments $[14,15]$ were recently replaced by new custom-made cryostats based on closed-cycle cryocoolers (SHI RDK-101E) [22]. A typical procedure included slow controlled deposition of a gaseous mixture onto a cooled support (KBr plate for IR studies of sapphire rod for EPR measurements) followed by irradiation with fast electrons or $\mathrm{x}$ rays through a special aluminum foil window. The deposition temperature was adjusted to yield monomeric samples (with low concentration of associates) of reasonable optical quality. For small molecules good results were obtained if depositing the matrix at ca. $30 \mathrm{~K}$, while higher temperatures ( 45 to $50 \mathrm{~K}$ ) were sometimes used for larger molecules at high dilution. The irradiation and spectral 
measurements were carried typically at $16 \mathrm{~K}$ (in some cases, the EPR spectra were also recorded at higher temperatures to follow reversible dynamics of the radiationinduced radicals).

\subsection{Fast electron irradiation vs. $x$-ray irradiation}

Two kinds of ionizing radiations were used in experiments. Early studies were carried out with fast electrons $(\sim 1 \mathrm{MeV})$ produced by a Van-de-Graaf type accelerator EG-2.5. Other experiments were performed using $x$ rays generated by a $5-\mathrm{BKhV}-6(\mathrm{~W})$ tube with a tungsten anode (typical voltage $33 \mathrm{keV}$, anode current $70 \mathrm{~mA}$ ). In fact, in both cases, the radiation-chemical effects are mostly induced by secondary electrons with relatively low energies, so there is no principal difference in chemistry, independent of the irradiation type. Indeed, qualitative results were found to be rather similar for all the systems studied. Meanwhile, significant quantitative difference may arise from different mechanisms of primary physical interactions. The basic mechanism for fast electrons is inelastic interaction with bound electrons of the medium resulting in ionization and electron excitation. There is no strong effect of the absorber nature and, in the case of $1-\mathrm{MeV}$ electrons, the absorbed dose is distributed nearly uniformly within the deposited layer $(\leq 0.5 \mathrm{~mm})$ and it can be easily determined using a monitor electrode as described previously [15]. Quantitative dosimetry is problematic for x-ray radiolysis because of wide energy spectrum and inhomogeneous dose distribution. Meanwhile, in the case of x-ray photons with the energy below $50 \mathrm{keV}$, the principal mechanism is photoeffect (photoelectric absorption) and the interaction cross-section increases sharply for heavy elements. In particular, in the case of xenon, the mass absorption coefficients are $54.2,24.7$, and $8.0 \mathrm{~cm}^{2} / \mathrm{g}$ for the photon energies of 15,20 , and $30 \mathrm{keV}$, respectively [34]. These values are quite high, which implies high absorbed doses upon x-ray irradiation of xenon matrices. In practice this means high efficiency of radiolysis in the given irradiation geometry. Indeed, $x$-ray irradiation of xenon matrices for 30-60 min typically produced large effect, comparable with that achieved upon fast electron irradiation at rather high doses as shown in Fig. 1 (it was not the case for argon). On the other hand, taking an estimate of effective photon energy of $20 \mathrm{keV}$ (at $\mathrm{E}_{\max } \sim 30 \mathrm{keV}$ ), one can calculate that a $50 \%$-absorption occurs within the xenon layer of ca. $0.1 \mathrm{~mm}$, which is comparable to the layer thickness in our IR experiments and definitely less than that in the EPR experiments. Thus, an inhomogeneous distribution of the radiation-chemical effects may be significant, especially in the samples used for EPR studies. To avoid exhausting the guest molecules in the surface sample layer and to minimize secondary reactions, it was practicable to use short exposure time (30 min or less) in the EPR experiments, even though an overall consumption of the guest molecules monitored by IR spectroscopy was not so high.
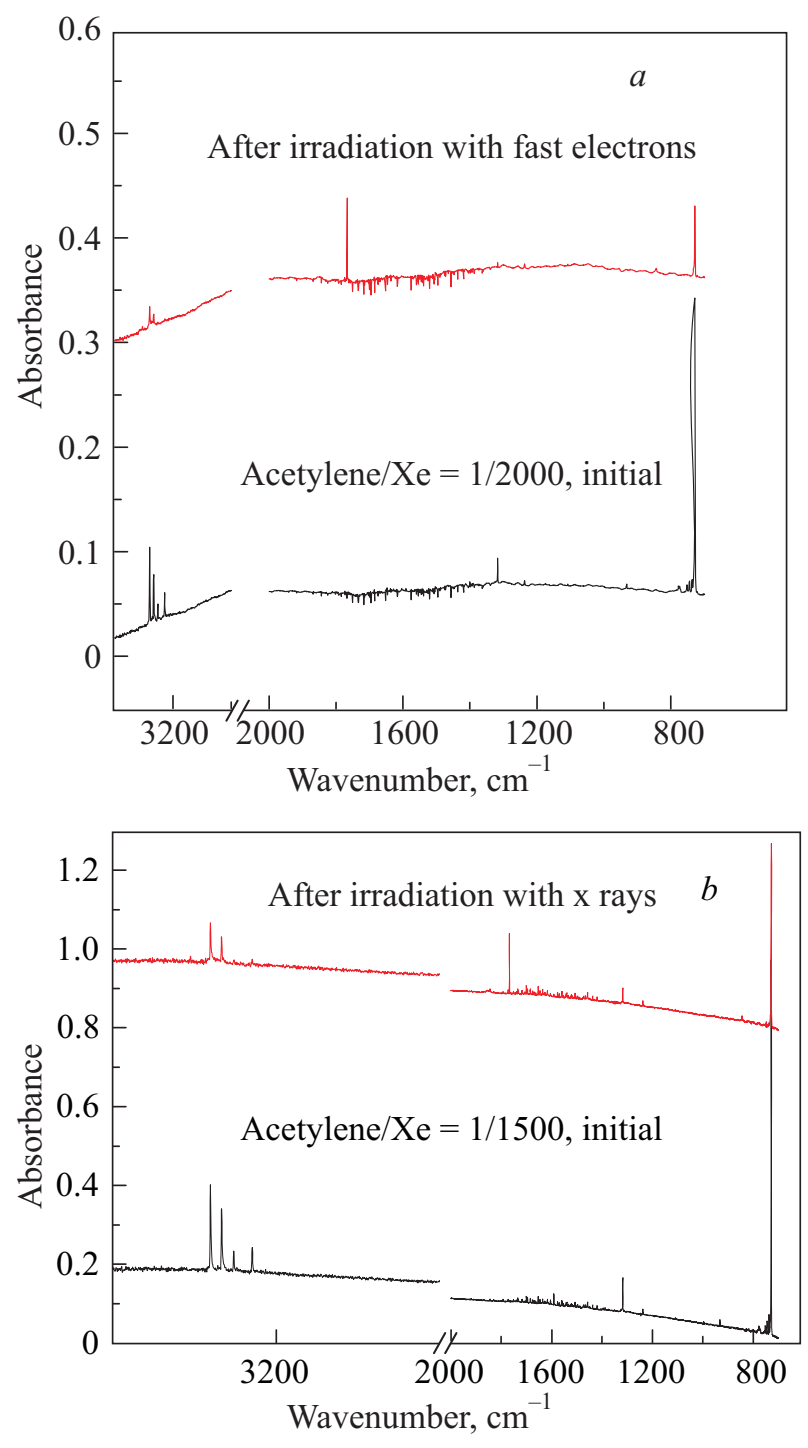

Fig. 1. The radiation-induced decomposition of acetylene in solid xenon under the action of fast electrons (absorbed dose: $80 \mathrm{kGy}$ ) (a) and $\mathrm{x}$ rays (60 min irradiation) (b) as revealed by IR spectroscopy.

\subsection{Experiments with monoisotopic and isotopically enriched xenon matrices}

As mentioned above, an important shortcoming of xenon matrix for EPR studies is concerned with high natural abundance of magnetic isotopes $\left({ }^{129} \mathrm{Xe}, I=1 / 2\right.$ and ${ }^{131} \mathrm{Xe}$, $I=3 / 2$ ), which affects strongly the EPR linewidth for trapped radical species. In order to overcome this limitation, we have suggested to use a specific monoisotopic xenon matrix $\left(99.4 \%\right.$ of $\left.{ }^{136} \mathrm{Xe}, I=0\right)[35,36]$. This approach is particularly useful for small radicals, which yield highresolution EPR spectra with narrow lines in a ${ }^{136}$ Xe matrix (actually, these spectra are rather similar to those recorded in an argon matrix, so a monoisotopic ${ }^{136} \mathrm{Xe}$ matrix may be described as "argon-like xenon" in terms of magnetic properties). An illustrative example is shown in Fig. 2.

In addition, we have used an isotopically enriched xenon matrix (86.2\% of ${ }^{129} \mathrm{Xe}$, no other magnetic isotopes) 


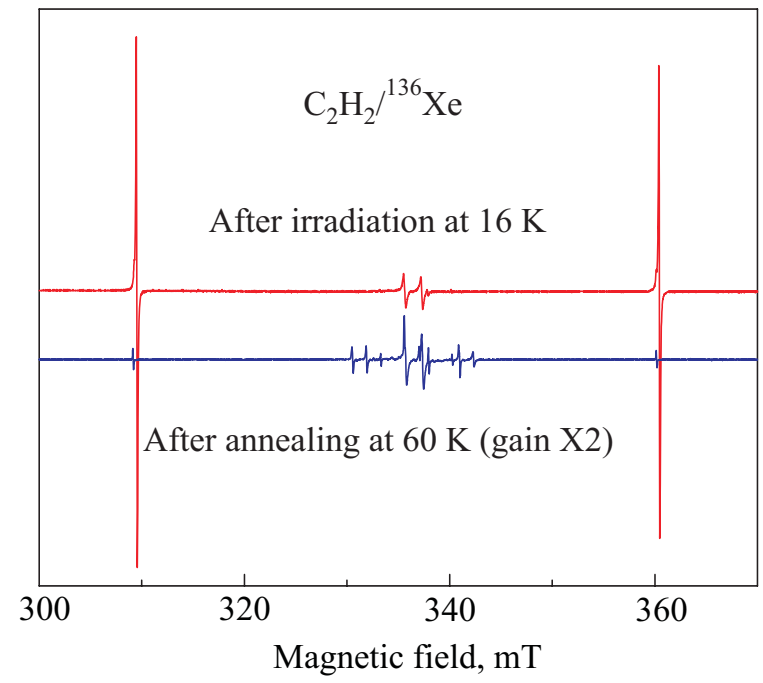

Fig. 2. EPR spectra of the system $\mathrm{C}_{2} \mathrm{H}_{2} /{ }^{136} \mathrm{Xe}=1 / 2000$ irradiated with fast electrons before and after annealing. The well-resolved signals from ethynyl radicals and vinyl radicals (after annealing) are clearly seen. A narrow doublet with very large splitting disappearing upon annealing is due to interstitially trapped $\mathrm{H}$ atoms in a ${ }^{136}$ Xe matrix (see Refs. 36, 37 for details).

and specially prepared mixtures (magnetically doped ${ }^{136} \mathrm{Xe}$ ). This approach made it possible to give an unequivocal assignment of the trapping site structure for $\mathrm{H}$ atoms produced in solid xenon [36].

Another application of monoisotopic xenon matrices for structural studies can be illustrated by observation of xenon isotopic shift in the IR spectra of xenon hydrides [37].

The xenon isotopes were obtained from State Research Center Kurchatov Institute (Moscow).

\section{Overwiew of results and discussion}

\subsection{Efficiency and mechanism of the radiation-induced degradation of guest molecules in solid xenon}

An overall efficiency of the total radiation-induced degradation of isolated molecules in a solid xenon matrix was estimated from IR spectroscopic studies of the samples irradiated with fast electrons $[13,15]$. According to these data, the radiation-chemical yields of consumption of guest species were found to be between 1 and 2.5 molecules per $100 \mathrm{eV}$ of energy absorbed by the matrix. In fact, this means that solid xenon is a very effective "transport system" for energy transfer to the isolated molecules at the dilution of 1:500 to 1:2000.

As stated above, positive hole transfer (reaction (2) in the scheme presented in Introduction) was found to be the basic mechanism for medium-size organic molecules with IP $\leq 10 \mathrm{eV}$. Thus, further transformations are determined by reactions of guest radical cations or excited states formed upon their recombination with electrons (5). It is worth noting that the properties of the recombination excited states may be different from those of optically attain- able excited states resulting from direct excitation upon photolysis and this may lead to different chemistry. An illustrative example is represented by benzene. The primary process upon irradiation of benzene in solid xenon is positive hole transfer resulting in formation of benzene radical cations, which are trapped in the presence of electron scavengers [38]. Meanwhile, in the absence of electron scavengers, we observed formation of nearly balanced amounts of phenyl radicals and trapped $\mathrm{H}$ atoms as confirmed by both EPR and IR spectroscopy [39]:

$$
\mathrm{C}_{6} \mathrm{H}_{6}{ }^{+}+\mathrm{e}^{-} \rightarrow \mathrm{C}_{6} \mathrm{H}_{6}{ }^{*} \rightarrow \mathrm{C}_{6} \mathrm{H}_{5}{ }^{\cdot}+\mathrm{H}^{\cdot} .
$$

This process is actually unknown in common photochemistry of benzene (optical excitation of benzene results mainly in formation of valence isomers [40]). A likely explanation may be concerned with population of high triplet states upon ion-electron recombination.

In the case of small guest molecules with relatively high IP (e.g., $\mathrm{H}_{2} \mathrm{O}, \mathrm{C}_{2} \mathrm{H}_{2}, \mathrm{CH}_{4}$ ), the situation is not so clear. Indeed, if the ionization energy of a guest molecule is above the energy of xenon mobile hole, the positive hole transfer (2) becomes unfavorable. Exact verification of this criterion is not straightforward, because comparison of the gas-phase IP values $\left(\mathrm{IP}_{g}\right)$ may be used only for rough estimates. The ionization energy in solid matrix $\mathrm{IP}_{\text {sol }}$ can be expressed as follows:

$$
\mathrm{IP}_{\mathrm{sol}}=\mathrm{IP}_{g}-P^{+}-V_{0} .
$$

Here $P^{+}$is vertical (electronic) polarization energy for positive ion formed upon ionization and $V_{0}$ is the energy of the bottom of conduction band for excess electron in a xenon crystal. As we are interested in the difference between $\mathrm{IP}_{\text {sol }}$ for xenon and guest molecule (IP gap), we should take into account the difference in the $P^{+}$values for xenon and guest cation ( $V_{0}$ is constant for a xenon matrix). $P^{+}$is inversely proportional to the cation radius. However, this term is not well defined, especially for complex non-spherical ions. In the case of $\mathrm{CH}_{4}$, the hole transfer from xenon definitely does not occur for energetic reasons, whereas there is some uncertainty about $\mathrm{H}_{2} \mathrm{O}$ and $\mathrm{C}_{2} \mathrm{H}_{2}$ (in both cases, $P^{+}$should be large enough). Most probably, the activation of these small molecules is due to exciton transfer, which results in formation of the corresponding neutral excited molecules dissociating to $\mathrm{H}$ and counterpart radicals.

\subsection{Matrix-induced and matrix-assisted reactions}

As mentioned above, some medium-size radical cations can be easily trapped in xenon and characterized by EPR [14-16]. However, in many cases, we failed to observe the primary species resulting from the guest molecules $\left(\mathrm{RH}^{+} \cdot\right)$ with low IP values (e.g., ethers and aldehydes), even in the presence of electron scavengers $[15,17,18]$. Instead of this, the EPR spectra revealed high yields of the corresponding radicals $\left(\mathrm{R}^{*}\right)$, which are expected to result from their deprotonation. It is worth noting that direct formation of these 
radicals through homolytic dissociation of neutral exited states (6) can be excluded, because the yield of trapped $\mathrm{H}$ atoms in the presence of electron scavengers is negligible. Thus, the most plausible explanation is prompt deprotonation of the primary radical cations to a xenon matrix, which can be formally written as follows:

$$
\left(\mathrm{RH}^{+}\right)+n \mathrm{Xe} \rightarrow \mathrm{R} \cdot+\mathrm{H}^{+} \mathrm{Xe}_{n} .
$$

Here $\mathrm{H}^{+} \mathrm{Xe}_{n}$ denotes a "solvated proton" in xenon. In fact, linear centrosymmetrical cations $\mathrm{XeH}^{+} \mathrm{Xe}(n=2)$ are well known due to their characteristic sign in the IR spectra (progression with the strongest band at $731 \mathrm{~cm}^{-1}$ ) [41,42]. The corresponding bands always appeared in our radiolytic experiments in xenon. It is worth noting that solvated protons are probably not mobile in solid xenon at low temperature [43].

The transformation described by reaction (9) may be defined as a matrix-induced deprotonation. This process may become favorable due to high acidity of the primary radical cations and appreciable polarizability and proton affinity for xenon (the latter value is as high as $5.2 \mathrm{eV}$ [44]). An indication of similar-type process was also found in krypton, but not in argon [17]. A remarkable feature of deprotonation is site selectivity, which correlates with spin density distribution in the radical cations. The effect of selective deprotonation correlating with the spin density distribution was first found by Toriyama et al. [45,46] for linear alkane radical cations trapped in zeolites and molecular matrices. Later it was recognized as a general rule for different-type radical cations [20]. In accordance with this rule, the experiments with complex guest molecules always revealed formation of only one dominating radical corresponding to deprotonation from the maximum spin density position $[17,18,20]$. It should be noted that this site selectivity is expected only for deprotonation and not for dissociation of neutral excited states or hydrogen abstraction reactions. An illustrative case is tetrahydrofuran (THF) $[18,20]$. According to the scheme (1)-(7), when THF is irradiated in a solid xenon matrix, the formation of primary radical cations due to hole transfer (2) is followed by recombination (5) and non-selective homolytic dissociation of neutral excited molecules (6). As a result, the EPR spectra show trapped hydrogen atoms and a mixture of two types of neutral radicals from THF (TYF-2-yl and THF-3-yl). Addition of an electron scavenger (Freon) leads to suppression of the ion recombination reaction (5) and the yield of trapped $\mathrm{H}$ atoms is dropped, at least, by an order of magnitude. However, the THF radical cations are probably too acidic to be trapped in xenon, so they undergo prompt deprotonation from the maximum spin density position to yield exclusively THF-2-yl radicals. This change in radical composition clearly shows the change in the mechanism of radical formation in the presence of electron scavengers and provides a strong support for the proposed scheme.

Nevertheless, direct experimental verification of proton transfer to a xenon matrix is still lacking. It is worth noting that most observations of this process are related to oxygen-containing molecules (particularly, ethers). In this case, an alternative explanation may be an intramolecular rearrangement occurring immediately after formation of the primary radical cation, e.g.:

$$
\mathrm{ROCH}_{2} \mathrm{OR}^{,+} \cdot(\mathrm{Xe}) \rightarrow \mathrm{R}\left(\mathrm{OH}^{+}\right) \cdot \mathrm{CHOR}^{\star} .
$$

A polarizable xenon environment facilitates the reaction due to significant lowering of the activation barrier, that is, xenon acts as a catalyst. Thus, reaction (10) may be defined as a matrix-assisted process. Theoretical consideration of such rearrangement for the primary methanol radical cation $\left(\mathrm{CH}_{3} \mathrm{OH}^{+\cdot} \rightarrow{ }^{\cdot} \mathrm{CH}_{2} \mathrm{OH}_{2}{ }^{+}\right)$carried out by Fridgen and Parnis [47], indeed, revealed a significant catalytic effect of a xenon atom. In fact, this process, formally described as an intramolecular $\mathrm{H}$ shift, bears some resemblance to deprotonation to xenon, because a weakly bound acidic proton of the radical cation forms strong complex with a xenon atom in the transition state. For this reason, one may expect similar selectivity rules for deprotonation and intramoleccular $\mathrm{H}$ shift. The product of rearrangement is a distonic radical cation with separated charge and spin. From experimental point of view, it is rather difficult to distinguish between (9) and (10), because EPR spectra of neutral radicals and distonic radical cations of similar structure are rather similar (in other words, the $\mathrm{OH}$ proton is virtually "silent" in the EPR spectra). Further experimental and theoretical effort are needed to identify unambiguously the mechanisms of matrix effects for radical cations in solid xenon, which may be of fundamental interest for understanding of the medium effects in the condensed-phase radiation chemistry.

\subsection{Production, distribution and reactions of hydrogen atoms}

As follows from scheme (1)-(7), the $\mathrm{H}$ atoms may result from any hydrogen guest containing molecule upon irradiation in a xenon matrix. Indeed, high yields of trapped $\mathrm{H}$ atoms were observed from different precursors $[8,9,14,23,32]$. A characteristic feature of the EPR spectra of trapped $\mathrm{H}$ atoms in solid xenon is extended, complex superhyperfine structure (SHFS) resulting from interaction of unpaired electron with the nuclei of magnetic isotopes $\left({ }^{129} \mathrm{Xe}\right.$ and $\left.{ }^{131} \mathrm{Xe}\right)$. This structure was detected in the pioneering work of Foner et al. [48] and reproduced in many other studies, independent of the generation method (photolysis or radiolysis) $[8,9,23,49-51]$. However, analysis of the structure pattern consisting of large number of anisotropic components in natural xenon was found to be quite complicated, so the trapping site nature for $\mathrm{H}$ atoms in solid xenon remained under discussion for a long time [49,50,53,54]. Using monoisotopic and isotopically doped xenon matrices, we have obtained conclusive evidence that more than $99 \%$ of radiolytically produced $\mathrm{H}$ atoms are trapped in nearly undistorted the octahedral $\left(O_{h}\right)$ sites and 
only a very minor fraction occupies substitutional sites in the fcc xenon lattice [36]. As mentioned above, the observed structure of the EPR spectra does not depend on the generation method, so this conclusion should be also valid for $\mathrm{H}$ atoms produced by photolysis.

Another hot topic concerning the production of $\mathrm{H}$ atoms in solid xenon deals with their spatial distribution, which is of interest for basic understanding of dynamics of hot species in the xenon lattice and of crucial significance for lowtemperature chemistry involving $\mathrm{H}$ atoms. The spatial distribution of $\mathrm{H}$ atoms produced by photodissociation in solid xenon is still under discussion. LaBrake et al. [55] suggested long travel distance (ca. $10 \mathrm{~nm}$ or more) for hot $\mathrm{H}$ atoms produced by a 193-nm photolysis of $\mathrm{HBr}$ in a xenon matrix, judging from low yields of trapped atoms. On the other hand, Pettersson et al. [56] presented evidences for local character of the primary photodissociation of $\mathrm{HI}$ in solid xenon (without any significant losses of $\mathrm{H}$ atoms) and proposed a mechanism, which could explain large separation between $\mathrm{H}$ and I atoms at higher doses due to secondary photoreactions. Regarding radiolytic production of $\mathrm{H}$ atoms, we were able to estimate a lower limit of an average distance between $\mathrm{H}$ atoms and radical counterparts (ca. $4 \mathrm{~nm}$ ) on the basis of the EPR linewidth observed in our experiments in a ${ }^{136} \mathrm{Xe}$ matrix [36]. This result indicates rather distant or essentially random distribution of the dissociation fragments. However, it is worth noting that this conclusion may be not directly applicable to photolysis because of different nature of the precursor excited states. Moreover, as will be shown in the next subsection, there are some evidences for different initial spatial distribution of $\mathrm{H}$ atoms generated by photolysis and radiolysis.

From chemical point of view, it is important that the interstitially trapped $\mathrm{H}$ atoms can be thermally released (mobilized) at around $40 \mathrm{~K}$. This annealing-induced long-range mobility (sometimes described as "global mobility") is well documented by various methods [9,23,29,57], and it can be used to study thermal reactivity of $\mathrm{H}$ atoms in solid xenon. Different-type reactions (hydrogen abstraction, addition, recombination) were found to occur upon annealing of irradiated xenon matrices. Meanwhile, probably, the most specific and unusual process is formation of xenon hydrides, which will be considered in more detail below.

\subsection{Radiation-chemical synthesis of xenon hydrides}

As stated in the Introduction, the formation of xenon hydrides of the HXeY type occurs due to thermal-induced mobility of hydrogen atoms and may be formally represented by the following scheme:

$$
\mathrm{HY}(\mathrm{Xe}) \rightarrow \mathrm{H}^{*}(\mathrm{Xe})+\mathrm{Y}^{\bullet}(\mathrm{Xe}) \rightarrow \mathrm{HXeY}+\mathrm{HXeH} .
$$

The parentheses here mean that the species are trapped in a xenon matrix. An opportunity to use high-energy radiation for preparation of xenon hydrides was demonstrated already in our early work [31]. A common advantage of this method (in comparison with photolysis) results from the fact that it is not limited by optical absorption spectrum of precursor, because virtually any type of molecule may give an excited state in the frame of "ionization-recombination" scheme described above. This is particularly useful for the so-called "poor absorbers". Indeed, for example, saturated hydrocarbons can be easily activated upon radiolysis in a xenon matrix. On the other hand, some limitations of the radiation-chemical method may arise from involvement of ionic channels, which yield different reaction products.

To this moment, we have observed ten xenon-hydrides in the radiation-chemical experiments: $\mathrm{HXeH}, \mathrm{HXeCCH}$,

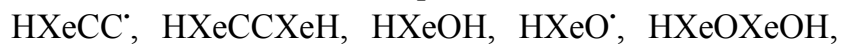
$\mathrm{HXeSH}, \mathrm{HXeBr}$, and $\mathrm{HXeCl}$ (all these species were identified previously in photochemical experiments, see Ref. 27 for review). Here we will focus on comparison between photolysis and radiolysis for preparation of $\mathrm{HXeY}$. First, we should note that the radiation-chemical technique is best suited for obtaining xenon dihydride $(\mathrm{HXeH})$, which can be produced with high yield from many kinds of hydrogenated precursors (HY or RH). According to the estimation from comparison of IR and EPR data [31], the formation of xenon dihydride is the major decay channel for $\mathrm{H}$ atoms generated upon radiolysis of xenon-hydrocarbon systems. This can be understood, if one takes into account a very diffuse distribution of $\mathrm{H}$ atoms upon radiolysis resulting in random recombination upon annealing, which is favorable for formation of $\mathrm{HXeH}$. On the other hand, photolysis may produce closer pairs H...Y (particularly, at low doses [56]), which results in increasing relative probability of formation of $\mathrm{HXeY}$. Second, we should comment the fact of relatively low yields of $\mathrm{HXeCC}^{*}$ radicals (and, correspondingly, $\mathrm{HXCCXeH}$ formed by their reactions) in the case of radiolysis [33], even at high doses. These species can be produced with high yields using photochemical dissociation of acetylene, especially at prolonged photolysis [58,59]. The precursor is a $\mathrm{Xe}-\mathrm{C}_{2}$ complex, which is easily detected by IR spectroscopy. Actually, this species is clearly seen in the radiation-chemical experiment already at relatively low doses. The limitation in its accumulation with increasing dose can be tentatively ascribed to effective reaction of $\mathrm{C}_{2}$ with excess electrons produced by radiolysis.

As a whole, more work should be done in order to elucidate detailed mechanisms of competing reaction channels for more reliable control of formation of the xenon hydrides using the radiation-chemical method. We believe that this method may be particularly promising for preparation of new hydrides from organic molecules, which are typically covalently bonded and often not very suitable for photochemical experiments.

\subsection{Production of oxygen atoms and ions}

There is a number of evidences for low-temperature chemical transformations involving oxygen atoms generated from appropriate precursors in solid noble gas matrices 
[60]. It is worth noting that, in the case of xenon, oxygen atoms become mobile at low temperatures (ca. 30-35 K, that is, before thermally induced global mobilization of $\mathrm{H}$ atoms) [61]. This annealing-induced mobility can be used for selective and specific chemical transformations, including preparation of novel unusual species. In particular, Khriachtchev et al. observed formation of $\mathrm{HXeO}^{*}$ radical [62] and $\mathrm{HXeOBr}$ molecule [63] due to thermal reactions of photolytically produced oxygen atoms. In the cited works the trapped oxygen atoms in solid xenon were obtained by two ways: (i) a two-stage dissociation of water molecules $\left(\mathrm{H}_{2} \mathrm{O} \rightarrow{ }^{\circ} \mathrm{OH} \rightarrow \mathrm{O}\right)$ occurring at high doses and (ii) photodissociation of $\mathrm{N}_{2} \mathrm{O}$. In order to make a comparison between photolysis and radiolysis as possible sources of oxygen atoms in solid xenon, we have also used these two precursors.

As shown in Fig. 3, the first scheme (a two-stage dissociation of $\mathrm{H}_{2} \mathrm{O}$ ) works quite well: the IR spectrum obtained after annealing of $\mathrm{H}_{2} \mathrm{O} / \mathrm{Xe}$ sample irradiated with fast electrons to high dose reveals a well-defined band of the $\mathrm{HXeO}^{\circ}$ radical (maximum at $1466 \mathrm{~cm}^{-1}$ [62]). In the case of $\mathrm{N}_{2} \mathrm{O}$, one needs an additional source of $\mathrm{H}$ atoms. We have used several different hydrogenated molecules for this purpose. The common procedure was similar to that used in photochemical studies [62]: irradiation of the system $\mathrm{HY} / \mathrm{N}_{2} \mathrm{O} / \mathrm{Xe}$ at low temperature following by annealing at $35 \mathrm{~K}$ to mobilize selectively trapped $\mathrm{O}$ atoms. However, in contrast to photolysis, the radiation-chemical experiments yield virtually no $\mathrm{HXeO}^{\circ}$ radical (Fig. 4 shows an example with $\mathrm{HBr}$ as a source of $\mathrm{H}$ atoms). At first glance, this result looks surprising, because the IR spectra reveal very effective radiation-induced decomposition of $\mathrm{N}_{2} \mathrm{O}$ (the corresponding bands disappear nearly completely after $20 \mathrm{~min}$ of x-ray irradiation). Also, the formation of sufficiently large amounts of trapped $\mathrm{H}$ atoms was demonstrated by EPR. Accordingly, annealing results in formation of some $\mathrm{HXeBr}$ and $\mathrm{HXeH}$ (major formation of these xenon hydrides occurs at higher temperature, when global

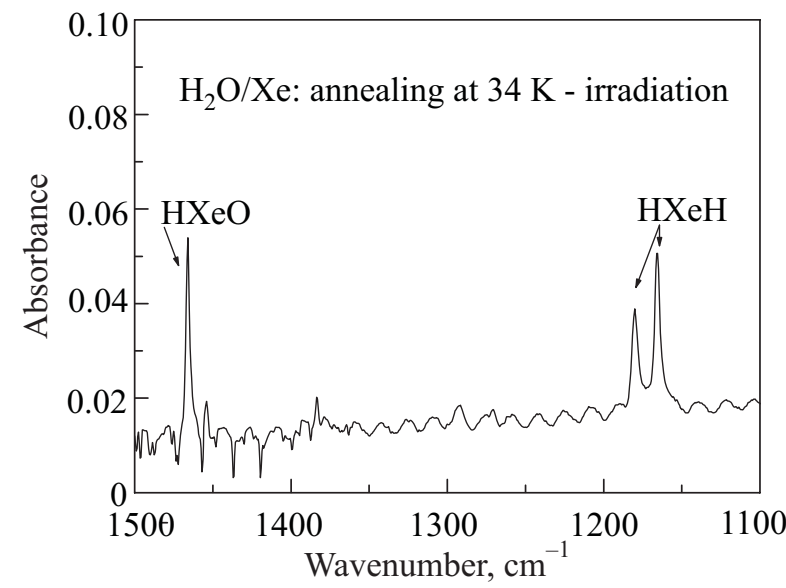

Fig. 3. The annealing-induced formation of the $\mathrm{HXeO}^{\circ}$ radical after irradiation of water in solid xenon $\left(\mathrm{H}_{2} \mathrm{O} / \mathrm{Xe}=1 / 2000\right)$.

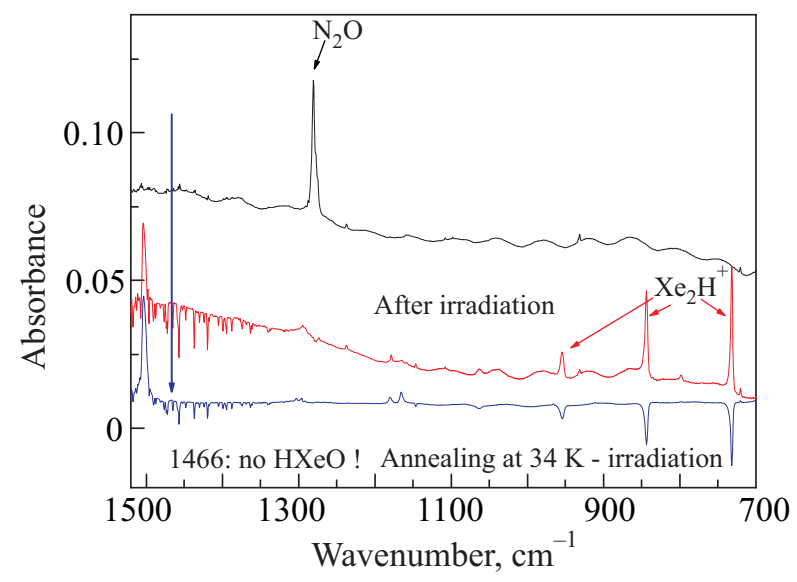

Fig. 4. Effect of $\mathrm{x}$-ray irradiation and subsequent annealing on the IR spectra of $\mathrm{HBr} / \mathrm{N}_{2} \mathrm{O} / \mathrm{Xe}$ system $(2 / 1 / 1500)$. The vertical arrow indicates the position of absorption from $\mathrm{HXeO}^{\circ}$ (see text for details).

mobility of $\mathrm{H}$ atoms is activated). The most plausible explanation of the absence (or very low yield) of the HXeO' radical is concerned with different mechanism of degradation of $\mathrm{N}_{2} \mathrm{O}$ molecules under irradiation. Indeed, in radiation chemistry it is quite common that $\mathrm{N}_{2} \mathrm{O}$ is a very good scavenger of excess electrons produced by radiolysis:

$$
\mathrm{e}^{-}+\mathrm{N}_{2} \mathrm{O} \rightarrow \mathrm{N}_{2}+\mathrm{O}^{-}
$$

These charged species are apparently immobile in a xenon matrix at low temperatures. We may suggest that the principal channel of radiolytic decomposition of $\mathrm{N}_{2} \mathrm{O}$ in solid xenon is formation of $\mathrm{O}^{-}$radical anions rather than neutral oxygen atoms (this conclusion are in accordance with preliminary EPR findings and with formation of large amount of $\mathrm{XeH}^{+} \mathrm{Xe}$, as clearly seen in Fig. 4 ). Thus, nitrous oxide is a not good source of oxygen atoms in the case of radiolysis; on the other hand, it is an excellent precursor of the $\mathrm{O}^{-}$radical anions, which can be of interest for further detailed structural and kinetic investigations.

\section{Conclusions}

As demonstrated in this mini-review, the studies of the radiation-induced transformations of guest molecules in solid xenon may provide important new information for various aspects of low-temperature chemistry and chemical physics. Actually, "the least noble" of noble gases provides a very interesting medium for model studies, which reveal rich and rather unusual chemistry. The chemical transformations are essentially controlled by a xenon matrix and may directly involve xenon as a reagent. We believe that our work in this area has led to some progress in understanding fundamentals of the solid-state radiation chemistry, trapping and dynamics of atoms and radicals, and preparation of novel unusual species. Meanwhile, there is still a number gaps and unresolved issues, which present new challenges for experiment and computations. 


\section{Acknowledgments}

The studies in our laboratory were continuously supported by the Division of Chemistry of The Russian Academy of Sciences under program No. 1.

1. Chemistry and Physics of Matrix-Isolated Species, L. Andrews and M. Moskovits (eds.), Elsevier, Amsterdam (1989).

2. Physics and Chemistry at Low Temperatures, L. Khriachtchev (ed.), Pan Stanford, Singapore (2011).

3. E.V. Savchenko, I.V. Khyzhniy, S.A. Uyutnov, G.B. Gumenchuk, A.N. Ponomaryov, and V.E. Bondybey, Nucl. Instr. Meth. Phys. Res. B268, 3239 (2010).

4. E.V. Savchenko, I.V. Khyzhniy, S.A. Uyutnov, G.B. Gumenchuk, A.N. Ponomaryov, and V.E. Bondybey, Fiz. Nizk. Temp. 35, 520 (2009) [Low Temp. Phys. 35, 409 (2009)].

5. I.V. Khyzhniy, S.A. Uyutnov, E.V. Savchenko, G.B. Gumenchuk, A.N. Ponomaryov, and V.E. Bondybey, Fiz. Nizk. Temp. 35, 433 (2009) [Low Temp. Phys. 35, 335 (2009)].

6. E.V. Savchenko, I.V. Khyzhniy, S.A. Uyutnov, G.B. Gumenchuk, A.N. Ponomaryov, M.K. Beyer, and V.E. Bondybey, J. Phys. Chem. A115, 7258 (2011).

7. W.V. Bouldin and W. Gordy, Phys. Rev. 135, A806 (1964).

8. D. Bhattacharya and J.E. Willard, J. Phys. Chem. 85, 154 (1981).

9. H. Muto, K. Toriyama, K. Nunome, and M. Iwasaki, Radiat. Phys. Chem. 19, 201 (1982).

10. K. Gotoh, T. Miyazaki, K. Fueki, and K.-P. Lee, Radiat. Phys. Chem. 30, 89 (1987).

11. L.B. Knight, Acc. Chem. Res. 19, 313 (1986).

12. K. Komaguchi, K. Nomura, and M. Shiotani, J. Phys. Chem. A111, 726 (2007).

13. V.I. Feldman, F.F. Sukhov, N.A. Slovokhotova, and V.P. Bazov, Radiat. Phys. Chem. 48, 261 (1996).

14. V.I. Feldman, Acta Chem. Scand. 51, 181 (1997).

15. V.I. Feldman, F.F. Sukhov, N.S. Nekhoroshev, V.K. Ivanchenko, and N.A. Shmakova, Khim. Vys. Energ. 32, 18 (1998) [High Energy Chem. 32, 15 (1998)].

16. V.I. Feldman, Radiat. Phys. Chem. 55, 565 (1999).

17. V.I. Feldman, F.F. Sukhov, A.Yu. Orlov, and N.A. Shmakova, J. Phys. Chem. A104, 3792 (2000).

18. V.I. Feldman, F.F. Sukhov, A.Yu. Orlov, and N.A. Shmakova, Khim. Vys. Energ. 35, 352 (2001) [High Energy Chem. 35, 319 (2001)].

19. V. Feldman, F. Sukhov, A. Orlov, and I. Tyulpina, Phys. Chem. Chem. Phys. 5, 1769 (2003).

20. V. Feldman, In: EPR of Free Radicals in Solids. Trends in Methods and Applications, A. Lund and M. Shiotani (eds.), Kluwer, Dordrecht (2003), p. 363.

21. V.I. Feldman, F.F. Sukhov, A.Yu. Orlov, I.V. Tyulpina, and V.K. Ivanchenko, Radiat. Phys. Chem. 75, 106 (2006).

22. A.V. Kobzarenko, F.F. Sukhov, A.Yu. Orlov, G.V. Kovalev, I.A. Baranova, and V.I. Feldman, Radiat. Phys. Chem. (2012); http://dx.doi.org/10.1016/j.radphyschem.2012.01.004.

23. V.I. Feldman, F.F. Sukhov, and A.Yu. Orlov, Chem. Phys. Lett. 280, 507 (1997).
24. T. Bally. In: Radical Ionic Systems. Properties in Condensed Phases, A. Lund and M. Shiotani (eds.), Kluwer, Dordrecht (1991), p. 3.

25. T. Bally, E. Haserlbach, S. Nitsche, and K. Roth, Tetrahedron 42, 6325 (1986).

26. M. Pettersson, J. Lundell, and M. Räsänen, J. Chem. Phys. 102, 6423 (1995).

27. L. Khriachtchev, M. Räsänen, and R.B. Gerber, Acc. Chem. Res. 42, 183 (2009).

28. M. Pettersson, J. Nieminen, L. Khriachtchev, and M. Räsänen, J. Chem. Phys. 107, 8423 (1997).

29. L. Khriachtchev, H. Tanskanen, M. Pettersson, M. Räsänen, V. Feldman, F. Sukhov, A. Orlov, and A.F. Shestakov, J. Chem. Phys. 116, 5708 (2002).

30. J. Lundell, L. Khriachtchev, M. Pettersson, and M. Räsänen, Fiz. Nizk. Temp. 26, 923 (2000) [Low Temp. Phys. 26, 680 (2000)].

31. V.I. Feldman and F.F. Sukhov, Chem. Phys. Lett. 255, 425 (1996).

32. V.I. Feldman, F.F. Sukhov, A.Yu. Orlov, and I.V. Tyulpina, J. Am. Chem. Soc. 125, 4698 (2003).

33. V.I. Feldman, F.F. Sukhov, A.Yu. Orlov, I.V. Tyuilpina, E.A. Logacheva, and D.A. Tyurin, Russ. Chem. Bul. 54, 1458 (2005).

34. J.H. Hubbel and S.M. Settler, Tables of x-ray mass attenuation coefficients and mass energy-absorption coefficients from $1 \mathrm{keV}$ to $20 \mathrm{MeV}$ for elements $Z=1$ to 92 and 48 additional substances of dosimetric interest (1996); http://www.nist.gov/pml/data/xraycoef.

35. V.I. Feldman, F.F. Sukhov, A.Yu. Orlov, and I.V. Tyulpina, Mendeleev Communs. 18, 121 (2008).

36. V.I. Feldman, F.F. Sukhov, and A.Yu. Orlov, J. Chem. Phys. 128, 214511 (2008).

37. V.I. Feldman, A.V. Kobzarenko, I.A. Baranova, A.V. Danchenko, F.F. Sukhov, E. Tsivion, and R.B. Gerber, J. Chem. Phys. 131, 151101 (2009).

38. V.I. Feldman, F. Sukhov, A. Orlov, R. Kadam, Y. Itagaki, and A. Lund, Phys. Chem. Chem. Phys. 2, 29 (2000).

39. V.I. Feldman, F.F. Sukhov, E.A. Logacheva, A.Yu. Orlov, I.V. Tyulpina, and D.A. Tyurin, Chem. Phys. Lett. 437, 207 (2007).

40. R. Barltrope and J. Coyle, Excited States in Organic Chemistry, John Wiley \& Sons, London (1975).

41. A.A. Karatun, F.F. Sukhov, and N.A. Slovokhotova, Khim. Vys. Energ. 15, 471 (1981).

42. H. Kunttu, J. Seetula, M. Räsänen, and V.A. Apkarian. J. Chem. Phys. 96, 5630 (1992).

43. H. Tanskanen, L. Khriachtchev, A. Lignell, M. Räsänen, S. Johansson, I. Khyzhniy, and E. Savchenko, Phys. Chem. Chem. Phys. 10, 692 (2008).

44. NIST Chemistry Webbook. NIST Standard Reference Database Number 69 (2011). http://webbook.nist.gov/chemistry/

45. K. Toriyama, K. Nunome, and M. Iwasaki, J. Phys Chem. 90, 6836 (1986).

46. K. Toriyama, K. Nunome, and M. Iwasaki, J. Am. Chem. Soc. 109, 4496 (1987). 
47. T.D. Fridgen and J.M. Parnis, Int. J. Mass. Spectrom. 190/191, 181 (1999).

48. S.N. Foner, E.L. Cochran, W.A. Bowers, S.N. Foner, and C.K. Jen, J. Chem. Phys. 32, 963 (1960).

49. K. Kinugawa, T. Miyazaki, and H. Hase, J. Phys. Chem. 82, 1697 (1978).

50. J.R. Morton, K.F. Preston, S.J. Strach, F.J. Adrian, and A.N. Jette, J. Chem. Phys. 70, 2889 (1979).

51. H.F. Fenrick, D. Bhattacharya, and J.E. Willard, J. Phys. Chem. 85, 1324 (1981).

52. M. Iwasaki, K. Toriyama, H. Muto, K. Nunome, and M. Fukaya, J. Phys. Chem. 85, 1326 (1981).

53. T. Kiljunen, J. Eloranta, and H. Kunttu, J. Chem. Phys. 110, 11814 (1999).

54. J. Eloranta, K. Vaskonen, and H. Kunttu, J. Chem. Phys. 110, 7917 (1999).

55. D. LaBrake, E.T. Ryan, and E. Weitz, J. Chem. Phys. 102, 4112 (1995).
56. M. Pettersson, L. Khriachtchev, R.-J. Roozeman, and M. Räsänen, Chem. Phys. Lett. 323, 506 (2000).

57. J. Eberline and M. Creuzburg, J. Chem. Phys. 106, 2188 (1997).

58. L. Khriachtchev, H. Tanskanen, M. Pettersson, H. Kiljunen, and M. Räsänen, J. Am. Chem. Soc. 125, 4696 (2003).

59. H. Tanskanen, L. Khriachtchev, J. Lundell, and M. Räsänen, J. Chem. Phys. 121, 8291 (2004).

60. E.Y. Misochko, A.V. Akimov, and I.U. Goldshleger, Usp. Khim. 72, 262 (2003).

61. A.V. Danilychev and V.A. Apkarian, J. Chem. Phys. 100, 8617 (1993).

62. L. Khriachtchev, M. Pettersson, J. Lundell, H. Tanskanen, T. Kiviniemi, N. Runeberg, and M. Räsänen, J. Am. Chem. Soc. 125, 1454 (2003).

63. L. Khriachnchev, S. Tapio, A.V. Domanskaya, M. Räsänen, K. Isokoski, and J. Lundell, J. Chem. Phys. 134, 124307 (2011). 